\title{
Estudio epidemiológico de las intoxicaciones agudas atendidas en un hospital gallego entre 2005 y 2008
}

\section{Epidemiological study of acute poisoning cases treated at a Galician hospital between 2005 and 2008}

\author{
José Carlos de Miguel-Bouzas*; Eva Castro-Tubío*; \\ Ana María Bermejo-Barrerera ${ }^{* *}$; Purificación Fernández- \\ Gómez ${ }^{\star \star *}$; Juan CARlos Estévez-Núñez ${ }^{\star \star \star *}$; MaRía Jesús \\ TABERNERO-DUQUE ${ }^{\star * *}$
}

\author{
*Servicio de Farmacia. Hospital Povisa. \\ ${ }^{*}$ Central de Coordinación Urxencias Sanitarias de Galicia - 061. \\ ${ }^{* \star \star}$ Instituto de Medicina Legal. Facultad de Medicina. Universidade de \\ Santiago de Compostela. \\ ${ }^{\star \star \star \star}$ Departamento de Economía Cuantitativa. Universidade de Santiago de \\ Compostela \\ Enviar correspondencia a: \\ José Carlos de Miguel Bouzas. \\ Instituto de Medicina Legal. Facultad de Medicina. \\ C/ San Francisco s/n. 15782 Santiago de Compostela, España \\ Correo electrónico: charlidemiguel@hotmail.com.
}

\section{Resumen}

Se realizó un estudio descriptivo retrospectivo de las intoxicaciones agudas registradas en el Complexo Hospitalario de Pontevedra (CHOP) entre enero de 2005 y diciembre de 2008, para conocer el número y tipo de intoxicaciones atendidas, su distribución según el sexo y edad del paciente, cronología, tipo de agentes tóxicos implicados, intencionalidad, antecedentes, clínica, evolución, tratamiento y análisis toxicológico empleado en el diagnóstico. Mediante los programas informáticos Clínica e IANUS y la consulta de todas las historias clinicas en papel de los pacientes atendidos con sintomas de intoxicación, se registraron los datos necesarios. Se incluyeron un total de 1893 pacientes con una edad media de 35,6 \pm 17,6 años (66\% hombres). La mayor incidencia se alcanzó los sábados y domingos durante los meses de verano (junio, julio y agosto). Los agentes tóxicos más frecuentes fueron las drogas de abuso $(70,4 \%)$, destacando entre ellas el alcohol etílico (61\%), frecuentemente asociado al sexo masculino y a un elevado grado de dependencia. En segundo lugar se situaron las intoxicaciones medicamentosas, frecuentemente asociadas al sexo femenino y producidas, en un $73,2 \%$ de los casos, por benzodiacepinas. La mayoria de las intoxicaciones registradas fueron voluntarias y en el $18,8 \%$ hubo intencionalidad suicida. Ésta se asoció mayoritariamente al sexo femenino. Las manifestaciones neurológicas fueron las más frecuentes y la mortalidad tan solo fue del 0,2\%.

Palabras clave: Intoxicaciones agudas, epidemiología, Servicio de Urgencias.

\section{Abstract}

A descriptive retrospective study of acute intoxication cases registered at the Complexo Hospitalario de Pontevedra (CHOP) between January 2005 and December 2008 was performed to find out the number and types of poisoning cases treated, their distribution according to patient's sex and age, chronology, type of toxic agents involved, intentionality, history, symptoms, clinical development, treatment and toxicological analysis used for diagnosis. Data were recorded using Clinica and IANUS software and consulting all paper records of patients with symptoms of poisoning. Data from a total of 1893 patients with a mean age of $35.6 \pm 17.6$ years $(66 \%$ men) were included. Highest rates of poisoning were recorded on Saturdays and Sundays during the summer months (June, July and August). Drugs of abuse were the most common toxic agents (70.4\%), ethyl alcohol accounting for $61 \%$ of these cases, which often involved males and with people with high degrees of dependency. In second place was poisoning resulting from the abuse of medical drugs, more commonly associated with females, and involving benzodiazepines in $73.2 \%$ of cases. The majority of these intoxications were intentional, and suicide attempts accounted for $18.8 \%$. The problems most commonly resulting from the poisoning were neurological, and mortality rate was just $0.2 \%$.

Key words: Acute intoxication, epidemiology, emergency departments. 
E la actualidad, el problema social que constituyen las intoxicaciones agudas, implica aspectos económicos, socioculturales, demográficos y psicológicos, y lejos de resolverse, hace crecer día a dia sus repercusiones sanitarias (de la Fuente, Barrio, Vicente, Bravo y Santacreu, 1995; Torralba et al., 1996).

Si queremos tener una idea objetiva de la realidad toxicológica actual en nuestro entorno, es imprescindible la realización de estudios evolutivos que definan la tendencia del momento. Es importante conocer los agentes tóxicos más frecuentemente implicados y los posibles tratamientos para formar a los profesionales sanitarios y dotar de medios diagnósticos y terapéuticos a los servicios asistenciales de hospitales y Centros de Atención Primaria (Burillo-Putze, 2006; Fernández, Garcia, Romero y Marquina, 2008). Éstos deben actualizarse periódicamente en medios e instalaciones, ya que el patrón de las intoxicaciones varía continuamente y depende de la idiosincrasia de cada sociedad.

Existen diversos estudios epidemiológicos publicados en España a lo largo de los años en los que se estudian intoxicaciones agudas en general (Echarte et al., 2005; Ferrer et al., 2005; Rodríguez y Hinojal, 1994; Sola, 1996) y también otros que en concreto estudian un determinado tipo de intoxicaciones (sólo por drogas de abuso, sólo medicamentosas, sólo voluntarias, etc.) (Duce et al., 1998; Medina, Fuentes, Suárez, Arranz y Ochoa, 2008; Rey, 2009). Pero en la comunidad gallega existen muy pocos trabajos publicados al respecto (Fernández et al., 2003; Rey, 2009), y mucho menos, en el área sanitaria de Pontevedra (Fernández, Sertal, Bermejo y Tabernero, 2005).

Debido a esta falta de datos se ha planteado este trabajo con el objetivo de realizar un estudio descriptivo de las intoxicaciones agudas registradas en el área sanitaria del Complexo Hospitalario de Pontevedra (CHOP). Se pretende conocer el número y tipo de intoxicaciones, su distribución según el sexo y edad de los pacientes, cronología, tipo de tóxicos implicados, antecedentes, clínica, evolución y tratamiento. Estos datos serán analizados y comparados, en la medida de lo posible, con los obtenidos por otros autores.

\section{Método}

Los datos para este estudio han sido facilitados por el Servicio de Documentación Clínica del CHOP, que clasifica a todos los pacientes atendidos en el hospital en función del motivo de ingreso. En este caso, se consiguieron datos de los 1893 pacientes clasificados como "Intoxicación" en el motivo de ingreso entre 2005 y 2008. Posteriormente, mediante los programas informáticos Clínica e IANUS y la consulta de todas las historias clínicas en papel de los pacientes mencionados, se obtuvieron las variables consideradas de interés para este estudio: fecha y hora de intoxicación, de llegada al hospital, de atención médica y de activación del Servicio de Coordinación Urxencias Sanitarias de Galicia - 061 (servicio que coordina la asistencia de ambulancias en Galicia), servicio de entrada y servicio de atención, sexo, edad, agente tóxico consumido, intencionalidad, antecedentes, manifestaciones clínicas, evolución clínica, tratamiento, muestra analizada y fecha y hora de alta.

El CHOP es un complejo hospitalario formado por el Hospital Provincial y el Hospital Montecelo de Pontevedra. En total cuenta con 622 camas de hospitalización y su área sanitaria comprende un total de 228.981 habitantes distribuidos en 21 ayuntamientos.

Para el estudio de las sustancias tóxicas implicadas se consideró el resultado analítico (positivo, negativo o dudoso) proporcionado por el Servicio de Análisis Clínicos y el Servicio de Farmacia del $\mathrm{CHOP}$, pero la información contenida en el historial médico del paciente, escrita por el médico el día de la intoxicación y apoyada en el resultado analítico obtenido, fue lo que determinó el tipo de intoxicación que se registró. Para realizar las determinaciones de drogas de abuso en orina, el Servicio de Análisis Clínicos empleó una prueba llamada DRUG-CLIP TEST $10^{\circledR}$, un test inmunocromatográfico de flujo lateral que detecta 10 drogas de abuso y es distribuido por A. Menarini diagnostics. Para cuantificar paracetamol en sangre, empleó el equipo Dimension $R \times L^{\oplus}$ de Siemens, que utiliza una prueba de diagnóstico in vitro llamada ACTM basada en la hidrólisis enzimática del acetaminofeno $y$ en la determinación de p-aminofenol mediante colorimetría utilizando una técnica de punto final bicromática. Para cuantificar los niveles de carboxihemoglobina se empleó la técnica de la cooximetría en los gasómetros 865 de Bayer. Y por último, el Servicio de Farmacia empleó un inmunoanálisis de fluorescencia polarizada (TDx ${ }^{\circledR}$ System Abbott) con el cual se podía cuantificar la concentración en sangre de fármacos tales como antiepilépticos y digoxina. Debemos destacar que, salvo excepciones, en el CHOP no se emplea ninguna técnica analítica para determinar alcohol en fluidos biológicos. El diagnóstico de los pacientes intoxicados por alcohol se establece por criterio médico en función de la sintomatología que presenten, y así se hace constar en el historial médico.

En el análisis estadístico de las variables cuantitativas se han usado la $F$ de Snedecor para comparar varianzas y la $T$ de Student para comparar dos medidas con distribución normal. Por su parte, para analizar las variables cualitativas se han usado la prueba de Chi cuadrado para determinar la significación de la asociación entre variables categóricas y el Contraste de proporciones para comparar porcentajes entre distintas categorías y determinar si las diferencias eran estadisticamente significativas o fruto del azar. Se han considerado estadisticamente significativos los valores de $p<0,05$.

El procesamiento y análisis de los datos se realizó mediante el paquete estadístico SPSS $17.0^{\circledR}$.

\section{Resultados}

Durante los cuatro años en los que se realizó este estudio acudieron 1893 pacientes a los Servicios de Urgencias del CHOP por intoxicación, de los cuales 1757 fueron al Servicio de Urgencias del Hospital Montecelo y 136 al del Hospital 
Provincial. Esto supone un 0,51\% sobre el total de urgencias registradas en el complejo en ese periodo.

Se aprecia un predominio del sexo masculino en el total de intoxicaciones agudas, con un $66 \%$ de casos frente a un $34 \%$ en el caso de las mujeres. Las intoxicaciones producidas por drogas de abuso están muy asociadas al sexo masculino $(76,7 \%)$, mientras que las intoxicaciones exclusivamente medicamentosas son más frecuentes en el sexo femenino $(66,7 \%)$.

\section{Edad}

La edad media del total de pacientes intoxicados fue de 35,6 \pm 17,6 años (rango: 1 - 90 años), 37,8 \pm 17,5 años en el caso de los hombres y $31,3 \pm 17,0$ años en el caso de las mujeres. Además, el 60\% de las intoxicaciones registradas en mujeres se sitúa en el intervalo 16-40 años, mientras que en el caso de los hombres, ese mismo porcentaje se sitúa en el intervalo 20 - 50 años. Las medias de edad más bajas aparecen en las intoxicaciones producidas por medicamentos exclusivamente, por drogas ilegales y por el grupo de "otros agentes", mientras que las más altas corresponden a las intoxicaciones exclusivamente etílicas.

Los pacientes pediátricos (entre 0 y 15 años) constituyen el $8,1 \%$ de los casos, de los cuales, más del $58 \%$, se debe a niños menores de 5 años.

\section{Distribución temporal}

El intervalo horario de intoxicación más frecuente es el comprendido entre las $12: 00$ y las $23: 59$ horas $(66,9 \%$ de los casos en los que se conocía la hora de intoxicación, un 10,7\% del total de pacientes) y la franja horaria más frecuente en la que los pacientes Ilegaron al hospital fue de 20:00 a 3:59 horas (el 49\% del total). El 42,9\% de los pacientes que Ilegaron al hospital en ambulancia tardaron menos de 30 minutos y prácticamente el 100\% de los pacientes que llegaron al hospital en ambulancia sin pasar previamente por su Centro de Atención Primaria, lo hicieron en menos de 45 minutos. Por otra parte, el tiempo comprendido entre la hora en que el paciente llegó al hospital y la hora en que recibió el alta médica también fue muy variable, desde unos pocos minutos hasta varios meses (estancia media: 26 horas y 27 minutos \pm 101 horas y 11 minutos), pero podemos decir que en el 73,2\% de los casos este tiempo fue inferior a 12 horas. De hecho, la estancia media de los pacientes que no ingresaron en ningún servicio y sólo permanecieron en Urgencias fue de 8 horas y 5 minutos \pm 8 horas y 51 minutos.

La máxima incidencia en la distribución semanal fue alcanzada los sábados y domingos $(42,4 \%$ del total de pacientes). El 33,2\% de las intoxicaciones registradas estos dos dias fue debido a drogas de abuso, y el 30,3\%, a alcohol. Las intoxicaciones etílicas resultaron mayoritarias en domingo, día en que se produjeron el $29,5 \%$ de las mismas. Por su parte, los meses en los que se registraron un mayor número de intoxicaciones fueron los de verano: junio, julio y agosto, con 199, 204 y 198 casos registrados respectivamente.

\section{Agente causal}

La Tabla 1 refleja la distribución de los casos registrados en función del tipo de intoxicación. Los agentes tóxicos implicados con mayor frecuencia fueron los del grupo de drogas de abuso (70,4\% del total), donde predomina el alcohol, presente en el $86,6 \%$ de las intoxicaciones producidas por drogas de abuso y en el $61 \%$ del total de intoxicaciones. Como se puede apreciar en la Figura 1, por detrás del alcohol, destacan la cocaína y el cannabis, representando el 12,4\% y $12 \%$ respectivamente de las intoxicaciones producidas por drogas de abuso.

A continuación, aparecen las intoxicaciones medicamentosas como el segundo grupo más numeroso $(33,1 \%$ del total), en concreto, las benzodiacepinas estaban presentes en el $73,2 \%$ de los casos y los analgésicos en el 10,2\%. Por último, el grupo de "otros agentes", formado por sustancias tales como el monóxido de carbono o los productos de limpieza, sólo representaron el 6,4\% del total.

A pesar de que el 52,4\% de las intoxicaciones registradas están producidas por una única droga, se han encontrado numerosos casos de asociación entre dos sustancias. Así podemos destacar los 120 casos encontrados de asociación entre alcohol y benzodiacepinas, los 83 de alcohol y cannabis, los 78 de alcohol y cocaína, los 80 de cannabis y benzodiacepinas, los 66 de cannabis y cocaína o los 42 de heroína y cocaína.

Tabla 1. Tipo de intoxicación

\begin{tabular}{lcc}
\hline & No CASOS & \% TOTAL \\
\hline Drogas de abuso & 1333 & 70,4 \\
Alcohol & 1154 & 61,0 \\
Alcohol exclusivamente & 921 & 48,6 \\
Medicamentosas & 626 & 33,1 \\
Exclusivamente medicamentosas & 366 & 19,3 \\
Otros agentes & 122 & 6,4 \\
Sin determinar & 72 & 3,8 \\
\hline
\end{tabular}

\section{Intencionalidad de la intoxicación}

La intención recreativa es mayoritaria con un $32,7 \%$ de frecuencia. A continuación destaca la "dependencia", representando el $29,2 \%$ de los casos, lo que denota el elevado número de personas alcohólicas o dependientes de alguna otra droga. En tercer lugar aparece la intención suicida con un $18,8 \%$ de frecuencia y en cuarto lugar, la involuntaria, con un 9,5\%. Como muestra la Tabla 2, las intencionalidades "dependencia" y "recreativa" están claramente ligadas al sexo masculino, mientras que la suicida es significativamente mayor en mujeres que en hombres.

El 65\% de las intoxicaciones en que están implicados medicamentos únicamente (intoxicaciones exclusivamente medicamentosas) tienen su origen en un intento de suicidio, y en las intoxicaciones producidas por el grupo de "otros agentes" predomina el motivo involuntario, con más de un $85 \%$ de los casos. El 56\% de los pacientes con intencionalidad suicida tenían entre 21 y 40 años y en el intervalo 5 - 15 años el número de intoxicaciones con intención recreativa 
superó al de intoxicaciones involuntarias, las dos intencionalidades mayoritarias en este grupo de edad.

Tabla 2. Relación Intencionalidad - Sexo

\begin{tabular}{|c|c|c|c|c|c|}
\hline & \multicolumn{2}{|c|}{ Hombre } & \multicolumn{2}{|c|}{ Mujer } & \multirow{2}{*}{$\begin{array}{c}\text { Razón } \\
\text { hombre/mujer }\end{array}$} \\
\hline & $\mathrm{N}^{\circ}$ & $\%$ & $\mathrm{~N}^{\circ}$ & $\%$ & \\
\hline Voluntarias & 1052 & 84,2 & 476 & 73,7 & 2,2 \\
\hline Recreativas & 447 & 35,8 & 173 & 26,7 & 2,6 \\
\hline Suicidas & 110 & 8,8 & 246 & 38,2 & 0,4 \\
\hline Dependencia & 495 & 39,6 & 57 & 8,8 & 8,7 \\
\hline Involuntarias & 95 & 7,6 & 85 & 13,2 & 1,1 \\
\hline Desconocidas & 79 & 6,3 & 46 & 7,1 & 1,7 \\
\hline Otras & 23 & 1,9 & 37 & 6 & 0,6 \\
\hline
\end{tabular}

\section{Antecedentes}

Hay evidencia de antecedentes psiquiátricos en el 42,6\% de los casos y de alcoholismo diagnosticado en el 25,8\%. En las intoxicaciones medicamentosas destaca como antecedente más frecuente $(63,3 \%)$ el hecho de que el paciente, en el momento de la intoxicación, se encontrase a tratamiento con psicofármacos y también los antecedentes psiquiátricos $(61,2 \%)$. En las intoxicaciones producidas por alcohol exclusivamente destacan como antecedentes el hecho de que los pacientes fueran reincidentes $(45,6 \%)$, los casos de alcoholismo crónico $(45,1 \%)$ y los antecedentes psiquiátricos $(35,9 \%)$. En las intoxicaciones producidas por drogas ilegales tienen mayor incidencia los pacientes con antecedentes psiquiátricos $(50,8 \%)$, los reincidentes $(45,9 \%)$ y también es bastante frecuente $(44,3 \%)$ que estos pacientes intoxicados sean adictos a drogas por vía parenteral (ADVP).

Existe un predominio de varones en los casos de alcoholismo crónico (quintuplica al número registrado de mujeres), de reincidencia, de ADVP y de tratamientos con metadona; mientras que en las mujeres predominan los antecedentes psiquiátricos y el estar a tratamiento con psicofármacos en el momento de la intoxicación (Figura 2).

\section{Manifestaciones clínicas}

El 93\% de los pacientes intoxicados presentó algún tipo de manifestación clínica. Como se observa en la Figura 3, las manifestaciones neurológicas fueron las más frecuentes en el total de intoxicaciones registradas $(97,1 \%$ del total). En segundo lugar, y a mucha distancia, se encontraron las manifestaciones digestivas (14,8\% del total de intoxicaciones). El $84 \%$ de los pacientes con sintomatología digestiva también presentó sintomatología neurológica, 226 pacientes, la combinación más frecuente.

Las manifestaciones neurológicas fueron mayoritarias en ambos sexos y en todos los intervalos etarios. Se alcanzó el porcentaje más alto en el intervalo 41 - 50 años (95,2\%). Por su parte, en el intervalo 5 - 20 años es donde se alcanzó el porcentaje más alto de manifestaciones digestivas $(61,4 \%)$.

También se registró el número de pacientes atendidos en el hospital por intoxicación que se habian producido algún

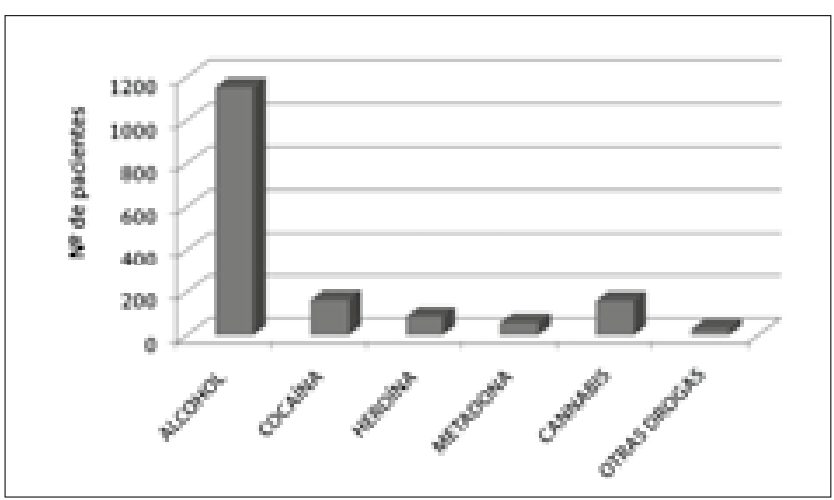

Figura 1. Drogas de abuso implicadas

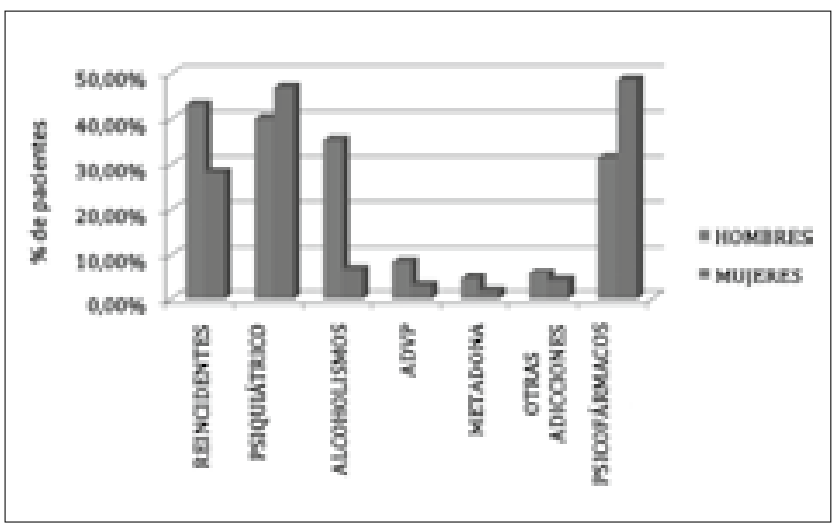

Figura 2. Antecedentes / Sexo

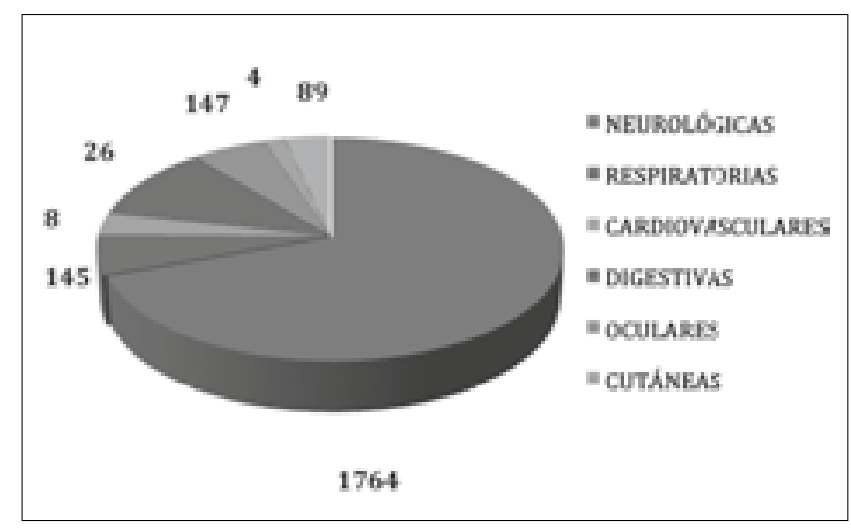

Figura 3. Manifestaciones clínicas

tipo de lesión traumatológica o herida propiciada por la pérdida de facultades tras la intoxicación, aumentando su estancia hospitalaria (203 pacientes). Este dato se vio que fue muy superior en el caso de los hombres que en el de las mujeres, apreciándose diferencias entre ambos $(p=.001)$.

\section{Tratamientos administrados}

El tratamiento empleado más frecuentemente fue el Ilamado inespecífico, en el 59,2\% de los pacientes. En segundo lugar, destaca la tiamina $(37,7 \%)$ (en ocasiones asociada a piridoxina), indicada para prevenir la encefalopatía de Wernicke y el sindrome de Korsakoff en las intoxicaciones por alcohol en pacientes alcohólicos; este tratamiento lo 
denominaremos específico para alcohol. A continuación se encuentran el lavado gástrico y el carbón activo, empleados en el $17 \%$ y el $14,8 \%$ respectivamente. Según el tipo de intoxicación, en las producidas por drogas de abuso los tratamientos más frecuentes fueron el inespecífico $(64,6 \%)$ y el específico para alcohol $(52,1 \%)$; en las medicamentosas presentó una incidencia máxima el lavado gástrico (47\%) con o sin carbón activo y el uso de medicación inespecifica $(51,6 \%)$; y en los casos relacionados con drogas ilegales y con "otros agentes", el tratamiento más frecuente fue el inespecífico (73,8\% y $60,7 \%$ respectivamente).

\section{Evolución de los pacientes}

La mayoría de los pacientes intoxicados evolucionaron satisfactoriamente. En el 74,3\% de los casos se prescribió el alta médica, el 10,7\% abandonó el hospital por iniciativa propia antes de recibir el alta $(6,2 \%$ se fugaron y $4,5 \%$ solicitaron el alta voluntaria), el 9,2\% requirió ingreso en algún servicio del hospital, y se produjo la muerte por intoxicación sólo en el 0,2\% de los casos, asociándose ésta a la dependencia a drogas de abuso, principalmente al alcohol. Los ingresos hospitalarios fueron más frecuentes en las intoxicaciones medicamentosas y en el $47,5 \%$ de los mismos estaban presentes las benzodiacepinas.

Por su relevancia clínica destacamos a los 35 pacientes $(1,8 \%$ del total) que ingresaron en la $\mathrm{UCl}$, y que suponen un 20,1\% del total de ingresos hospitalarios. Todos eran mayores de 15 años y un 60\% hombres. Los agentes tóxicos empleados con más frecuencia por estos pacientes fueron las drogas ilegales, la intencionalidad mayoritaria fue la suicida y el 51,4\% ingresó inconsciente en el servicio.

\section{Muestras biológicas}

La muestra biológica analizada con mayor frecuencia fue la orina (716 casos), seguida de la sangre (54 casos). No se utilizó ninguna prueba analítica para diagnosticar las intoxicaciones producidas por alcohol, tan solo se empleó la clínica del paciente. De las 771 pruebas analíticas realizadas resultaron positivas el 65\%, negativas el $31,1 \%$ y dudosas el $3,9 \%$. Las primeras fueron más frecuentes en las intoxicaciones medicamentosas.

\section{Discusión}

El porcentaje de intoxicaciones registradas en el Servicio de Urgencias del CHOP es similar a los publicados por otros autores (Burillo-Putze et al., 2003; Fernández et al., 2003; Fernández et al. 2008; Sola, 1996). A pesar de ello, es posible que haya habido pacientes intoxicados que en el momento de entrada en el hospital hayan sido clasificados bajo un epígrafe distinto al de "Intoxicación" y por tanto no hayan sido incluidos en el estudio. Se confirma el predominio del sexo masculino en el total de intoxicaciones agudas (Fernández et al., 2003; Ferrer et al., 2005; da Silva et al., 2010) y en las intoxicaciones por drogas de abuso; y el de las mujeres en las intoxicaciones medicamentosas (Amigo-Tadín, Nogué-Xarau y Miró-Andreu, 2010; Fernández et al., 2010; Medina et al., 2008; Sola, 1996).

Se aprecia un incremento en la media de edad de los pacientes intoxicados en la última década (Burillo-Putze et al., 2003; García-Baró et al., 2005; Fernández et al., 2008), siendo significativamente mayor la edad encontrada en hombres que en mujeres $(p=.000)$. Las intoxicaciones producidas por alcohol se asocian con una media de edad mayor, al igual que el alcoholismo crónico, lo que define el perfil típico del paciente que presenta esta patología. Por otra parte, las intoxicaciones producidas por drogas ilegales y las medicamentosas se asocian a las medias de edad más bajas, lo que también define al perfil del paciente consumidor de drogas ilegales (Fernández et al., 2003; Sola, 1996) y al perfil de paciente que consume medicamentos con intencionalidad suicida (Hermida, 1997).

No se ha estudiado el intervalo asistencial ya que la hora de intoxicación sólo pudo ser registrada en un porcentaje muy bajo de pacientes y no es valorable. Pero la franja horaria más frecuente en la que los pacientes acudieron al hospital tras una intoxicación (entre las 12:00 horas y las 23:59 horas) coincide con la publicada en otras series (Fernández et al., 2003; Rodríguez et al., 1994; Sola, 1996).

El tiempo medio de estancia de los pacientes en el hospital tiene una desviación estándar tan elevada que nos da una idea de la heterogeneidad de este tipo de pacientes, ya que en unos casos simplemente es necesario tratar los sintomas de la intoxicación de forma puntual, pero en otros se necesita paliar secuelas que ha dejado la intoxicación o bien tratar aspectos psiquiátricos que llevaron al paciente a intoxicarse de forma voluntaria.

La distribución anual y semanal de las intoxicaciones registradas fue la esperada tal y como apuntan varios autores, ya que el consumo de drogas de abuso se asocia habitualmente a momentos de ocio tales como los fines de semana (Burillo-Putze et al., 2003; Echarte et al., 2005; Fernández et al., 2003) y los meses de verano (Fernández et al., 2008; Güloglu y Hamdi, 2005).

La distribución obtenida de los distintos tipos de intoxicación y el predominio del alcohol como agente tóxico mayoritario concuerda a grandes rasgos con lo publicado por otros autores (Burillo-Putze et al., 2003; Fernández et al., 2003; Fernández et al., 2008; Ferrer et al., 2005) y nos recuerda que el elevado consumo de esta sustancia, muy aceptada socialmente en nuestro entorno, conlleva grandes repercusiones de tipo social y sanitario. Las benzodiazepinas por su parte, se consolidan como el medicamento más frecuentemente encontrado en las intoxicaciones, aumentando su frecuencia a medida que pasan los años (Duce et al., 1998; Fernández et al., 2005; Burillo-Putze et al., 2006; Fernández et al., 2008) en detrimento de otros fármacos como los analgésicos o los antidepresivos tricíclicos. Actualmente la prescripción de psicofármacos ha aumentado notablemente ya que son esenciales en el abordaje terapéutico de patologías cada día más frecuentes como la depresión y la ansiedad (Fernández et al., 2005), pero 
esta prescripción debería hacerse de forma muy controlada y prestando especial atención a los pacientes depresivos o con alto riesgo de depresión (Shah, Uren, Baker y Majeed, 2002), por riesgo a que éstos se intoxiquen con su propia medicación (Prescott y Highley, 1985). Por otra parte, en las intoxicaciones producidas por drogas de abuso, se ha visto como en la última década la cocaina ha adquirido un gran protagonismo (Burillo-Putze et al., 2006; Fernández et al., 2003; Fernández et al., 2008; García-Baró et al., 2005), mientras que el consumo de heroína ha disminuido sustancialmente. Según un informe publicado por la Xunta de Galicia en 2008, los modelos de policonsumo de drogas observados en Galicia son, además del uso concomitante de psicofármacos, la asociación de alcohol con psicoestimulantes (cocaína, cannabis y drogas recreativas) y la asociación de heroína con cocaína. En nuestra serie, un $47,7 \%$ de las intoxicaciones por heroína también dieron positivo a cocaína, resultado que está descrito como un patrón frecuente entre los consumidores de heroina (Barrio, Bravo y de la Fuente, 2000). Por otra parte, ya desde la década de los 90 se habla de la importancia de las asociaciones entre sustancias, principalmente cuando una de ellas es el alcohol (Stein, Bonanno, 0'Sullivan y Watchel, 1993), así, en nuestro estudio también se observa la frecuente asociación entre dos sustancias: alcohol y cocaina, alcohol y cannabis, y cocaina y cannabis, resultado que coincide con lo registrado en otros trabajos publicados en nuestro país (Galicia, Nogué, Sanjurjo y Miro, 2008; Caballero et al., 2008; Sanjurjo, Montori, Nogué, Sánchez y Munné, 2006).

Al igual que en otras series (Fernández et al., 2003; Medina et al., 2008), en nuestro trabajo también se aprecia como la mayoría de las intoxicaciones exclusivamente medicamentosas están motivadas por un intento de suicidio en el que, en la mayoría de los casos, están presentes las benzodiacepinas (Lambert, Manel, Bellou y El Kouch, 1997). A la vista de los resultados obtenidos, debería ser un motivo de reflexión y de preocupación el hecho de que la intencionalidad recreativa sea la mayoritaria en los pacientes en edad pediátrica (de 5 a 15 años), superando a la intencionalidad involuntaria, que a priori podría pensarse que sería mayoritaria.

El porcentaje de pacientes que presenta alcoholismo crónico concuerda con el de otros artículos publicados recientemente (Fernández et al., 2008), aunque duplica al encontrado en la década de los noventa en un área sanitaria próxima a la de nuestro estudio (Fernández et al., 2003). En ese mismo trabajo, encontramos que el porcentaje de pacientes con edades comprendidas entre los 31 y 40 años que estaban a tratamiento con psicofármacos era la mitad que en nuestro estudio (48,9\%), dato que nos habla del aumento de consumo de psicofármacos en España en la última década. Por último, mencionar que otros autores (Buckley et al., 1996) destacan que muchos pacientes estaban a tratamiento en el momento de la intoxicación, tal y como nosotros observamos en el 78,8\% de los intentos de suicidio.

Dado que el alcohol es el agente tóxico más frecuente en el total de intoxicaciones agudas, hemos considerado oportuno contemplar a la tiamina (ocasionalmente asociada a la piridoxina), tratamiento que se administra por protocolo en el Servicio de Urgencias del CHOP ante una intoxicación etílica grave en la que es difícil la anamnesis, como un tipo aparte del tratamiento inespecífico, denominándolo tratamiento específico para alcohol. Otros autores (Burillo-Putze et al., 2003; Echarte et al., 2005) han considerado este tratamiento como parte del tratamiento inespecífico y de ahí que hayan registrado valores de tratamiento inespecífico tan elevados. No obstante, en nuestro estudio el tratamiento inespecífico también resultó mayoritario, como en la mayoría de publicaciones.

Podemos afirmar que la intoxicación aguda no es una patología muy letal, tan sólo el $0,2 \%$ de los pacientes de nuestro estudio falleció por intoxicación, dato que viene a corroborar lo ya publicado por otros autores (Burillo-Putze et al., 2003; Echarte et al., 2005; Fernández et al., 2008; Ferrer et al., 2005) y que pone de manifiesto el problema social que existe en nuestro entorno con el alcoholismo, presente en al menos el 50\% de los pacientes fallecidos. En este trabajo hemos obtenido una tasa de ingreso en la $\mathrm{UCl}$ muy inferior a la encontrada en otras series (Burillo-Putze et al., 2003; GarcíaBaró et al., 2005; Henderson, Wright y Pond, 1993), aunque sí encontramos en común con otras publicaciones que los intentos de suicidio fueron la causa fundamental del ingreso en este servicio (Fernández et al., 2003; Gunawardana y Abeywarna, 1997). Podemos destacar también que 1 de cada 10 pacientes de nuestro estudio abandonó el hospital por voluntad propia y sin autorización médica (alta voluntaria y fuga), dato similar al publicado por otros autores (Burillo-Putze et al., 2003; GarcíaBaró et al., 2005); además esta tasa aumenta hasta un 14,2\% en las intoxicaciones por alcohol exclusivamente, lo que resulta previsible ya que la velocidad de metabolización del alcohol supera en muchos casos los tiempos de espera de los pacientes en los Servicios de Urgencias.

En el CHOP, exceptuando casos judiciales, no se analiza alcohol en sangre, sino que el diagnóstico de una intoxicación etílica se realiza únicamente en base al criterio clínico del médico y así lo hace constar en el historial del paciente. Esto puede parecer una limitación para el diagnóstico de este tipo de intoxicación, pero hay estudios que afirman que en el caso del alcohol no suele haber las discrepancias entre datos clínicos y analíticos propias de las drogas ilegales (Sanjurjo et al., 2005). Además, en los casos en los que el médico lo consideró oportuno, solicitó el análisis toxicológico de drogas de abuso en orina para confirmar si concomitantemente el paciente había consumido otras sustancias.

\section{Conflicto de intereses}

Los autores no tienen conflictos de intereses que declarar.

\section{Referencias}

Amigo-Tadín, M., Nogué-Xarau, S. y Miró-Andreu, O. (2010). Presentación clínica, actitud terapéutica y evolución de las intoxicaciones agudas tratadas con carbón activado: existen diferen- 
cias entre hombres y mujeres? Enfermería Clínica, 20, 273-279. doi:10.1016/j.enfcli.2010.06.003

Barrio, G., Bravo, M. J. y de la Fuente, L. (2000). Consumo de drogas ilegales en España: hacia una diversificación de los patrones de consumo y los problemas asociados. Enfermedades Emergentes, 2, 88-102.

Buckley, N. A., Dawson, A. H., Whyte, I. M., Hazell, P., Meza, A. y Britt, H. (1996). An analysis of age and gender influences on the relative risk for suicide and psychotropic drug selfpoisoning. Acta Psychiatrica Scandinavica, 93, 168-171. doi:10.1111/j.1600-0447.1996.tb10625.x.

Burillo-Putze, G., Munne, P., Dueñas, A., Pinillos, M. A., Naveiro, J. M., Cobo, J. y Alonso, J. (2003). National multicentre study of acute intoxication in emergency departments of Spain. European Journal of Emergency Medicine, 10, 101-104. doi:10.1097/00063110-200306000-00006.

Burillo-Putze, G., Pinillos-Echeverría, M. A., Jiménez-Lozano, M. A., Bajo-Bajo, A., Avilés-Amat J., Berruete-Cilveti, M... Dueñas-Laita, A. (2006). Organización y disponibilidad de recursos para la asistencia toxicológica en los servicios de urgencias de los hospitales españoles. Emergencias, 18, 219-228.

Caballero, P. J., Dorado, S., Díaz, A., Garcia, M. E., Yubero, L. y Torres, N. (2008). Vigilancia epidemiológica de la intoxicación aguda en el área sur de la Comunidad de Madrid: estudio VEIA 2004. Anales de Medicina Interna, 25, 262-268. doi:10.4321/S021271992008000600003 .

Duce, S., López, E., Navas, V., Piqueras, M., Jara, M., Servián, R... Abril, A. (1998). Intoxicaciones medicamentosas voluntarias atendidas en un Servicio de Urgencias. Emergencias, 10, 225-233.

Echarte, J. L., Iglesias, M. L., Hernández, E., García, L., Orriols, M., Villar, J...Skaf, E. (2005). Registro de las intoxicaciones agudas en un Servicio de Urgencias hospitalario. Toxicología, 22, 116.

Fernández, C., García, G., Romero, R. y Marquina, A. J. (2008). Intoxicaciones agudas en las urgencias extrahospitalarias. Emergencias, 20,328-331.

Fernández, P., Ortega, M., Bermejo, A. M., Tabernero, M. J., López-Rivadulla, M. y Concheiro, M. E. (2003). Intoxicaciones agudas en Santiago de Compostela en un periodo de cuatro años. Toxicología, 20, 216-220.

Fernández, P., Sertal, R., Bermejo, A. M. y Tabernero M. J. (2005). Intoxicaciones agudas por psicofármacos y drogas de abuso en Pontevedra durante el año 2001. Toxicología, 22, 37-40.

Ferrer, A., Royo, R., Rivas, M., Menao, S., Moreno, M. y Civeira, E. (2005). Perfil de las intoxicaciones agudas en el Servicio de Urgencias del Hospital Clínico de Zaragoza. Toxicología, 22, 121.

de la Fuente, L., Barrio, G., Vicente, J., Bravo, M. J. y Santacreu, J. (1995). The impact of drug-related deaths on mortality among young adults in Madrid. American Journal of Public Health, 85, 102-105. doi:10.2105/AJPH.85.1.102.

Galicia, M., Nogué, S., Sanjurjo, E. y Miro, O. (2008). Evolución de las consultas urgentes relacionadas con el consumo de cocaina durante el periodo 2002-2007. Emergencias, 20, 385-90.
Garcia-Baró, M., Lasarte-Turumbay, L., Prieto-Ferrer, M., Montiel, J. A., Lloret, J. y Marruecos, L. (2005). Intoxicaciones agudas del adulto. Epidemiología en un Servicio de Urgencias. Toxicología, 22, 119-20.

Güloglu C. y Hamdi, I. (2005). Acute poisoning cases admitted to a university hospital emergency department in Diyarbakir, Turkey. Human \& Experimental Toxicology, 24, 49-54. doi:10.1191/09603 27105ht499oa.

Gunawardana, R. H. y Abeywarna, C. (1997). Intensive care utilisation following attempted suicide through self-poisoning. Ceylon Medical Journal, 42, 18-20.

Henderson, A., Wright, M. y Pond, S. M. (1993). Experience with 732 acute overdose patients admitted to an intensive care unit over six years. Medical Journal of Australia, 158, 28-30.

Hermida, I. (1997). Estudio epidemiológico y características psicosociales de las intoxicaciones agudas en el ámbito hospitalario. Tesis Doctoral no publicada. Santiago de Compostela: Universidade de Santiago de Compostela.

Lambert, H., Manel, J., Bellou, A. y El Kouch, S. (1997). Morbidity and mortality from acute drug poisoning in France. La Revue $d u$ praticien, 47, 716-720.

Medina, L., Fuentes, M. E., Suárez, J. P., Arranz, M. I. y Ochoa, E. (2008). Epidemiología de las intoxicaciones medicamentosas durante un año en el Hospital Universitario Ramón y Cajal. Revista Clínica Española, 208, 432-436. doi:10.1157/13127603.

Rey, M. C. (2009). Epidemiología de las urgencias toxicológicas por drogas de abuso en el área sanitaria de Santiago de Compostela. Tesis Doctoral no publicada. Santiago de Compostela: Universidade de Santiago de Compostela.

Rodríguez, J. A. e Hinojal R. (1994). Intoxicaciones agudas: estudio epidemiológico retrospectivo en un área sanitaria asturiana 1985-1989. Toxicología, 11, 99-104.

Prescott, F. y Highley, M. (1985). Drugs prescribed for self poisoners. British Medical Journal, 290, 1633-1636. doi:10.1136/ bmj.290.6482.1633.

Sanjurjo, E., Cámara, M., Nogué, S., Negredo, M., Garcia, S., To-Figueras, J... de Pablo, J. (2005). Urgencias por consumo de drogas de abuso: confrontación entre los datos clínicos y analíticos. Emergencias, 17, 26-31.

Sanjurjo, E., Montori, E., Nogué, S., Sánchez, M. y Munné, P. (2006). Urgencias por cocaina: un problema emergente. Medicina Clínica, 126, 616-619. doi:10.1157/13087719.

Shah, R., Uren, Z., Baker, A. y Majeed, A. (2002). Trends in suicide from drug overdose in the elderly in England and Wales, 19931999. International Journal of Geriatric Psychiatry, 17, 416-421. doi:10.1002/gps.625.

da Silva, C., Rezende, N., Alves, R., Marangon, P., Carneiro, P. y Teixeira, M. (2010). Análise retrospectiva das intoxicações admitidas no hospital universitario da UFJF no periodo 2000-2004. Ciencia \& Saúde Coletiva, 15, 879-888.

Sola, J. L. (1996). Estudio epidemiológico de las intoxicaciones agudas en el Hospital Clínico Universitario de Zaragoza en el período 1990-1994. Tesis Doctoral no publicada. Zaragoza: Universidad de Zaragoza. 
Stein, M. D., Bonanno, J., O'Sullivan, P. S. y Watchel, T. J. (1993). Changes in the pattern of drug overdoses. Journal of General Internal Medicine, 8, 179-184. doi:10.1007/BF02599261.

Torralba, L., Brugal, M. T., Villalbi, J. R., Tortosa, M., Toribio, A. y Valverde, J. L. (1996). Mortality due to acute adverse drug reactions: opiates and cocaine in Barcelona 1989-1993. Addiction, 91, 419-426.

Xunta de Galicia (2008). 0 consumo de drogas en Galicia IX 2006. Consellería de Sanidade. Servizo Galego de Saúde. 